\title{
Multifractal analyses of human response time: potential pitfalls in the interpretation of results
}

\author{
Espen A. F. Ihlen * \\ Department of Neuroscience, Norwegian University of Science and Technology, Trondheim, Norway \\ *Correspondence: espen.ihlen@ntnu.no
}

Edited by:

José M. Medina, Universidad de Granada, Spain

Reviewed by:

Fred Hasselman, Radboud University Nijmegen, Netherlands

Helmut Ahammer, Medical University of Graz, Austria

Keywords: response times, $1 / f$ noise, multifractal, variability, long-range dependency, fractal

\section{INTRODUCTION}

Analyses of response time series have provided insight into mental organization and cognitive processes used in a wide variety of tasks such as simple reaction time, word naming, choice decision, visual search, memory search, and lexical decision (Gilden, 2001). One of the new and frequently used sets of analyses is the numerical definition of scale invariant structure of response time series, also called $1 / f$ fluctuations. Componentoriented theories suggest that this scale invariant structure originated from an idiosyncratic mechanism in the cognitive system, whereas interaction-oriented theories argue that scale invariant structure in response time series arises from selforganizing interaction between different sources and mechanisms (cf. Diniz et al., 2011). In this short commentary, new analyses of human response time called multifractal analyses will be introduced, and potential pitfalls of interpreting the results of these analyses will be discussed.

Multifractal analyses quantify the intermittent structure of response time series that are created by interactions between temporal scales of response series (Ihlen and Vereijken, 2010, 2013). Even though these analyses have been recently introduced in analysis of human behavior, their mathematical fundament of these analyses was introduced four decades ago (Yaglom, 1966; Mandelbrot, 1974). Typically, response time series with a large number of trials will contain intermittent periods with a higher number of slow response latencies than the rest of the response series (e.g., Holden et al.,
2009). These intermittent periods of slow response latencies might indicate shifts in the participant attention to the stimuli source or active periods of response error corrections (Ihlen and Vereijken, $2010,2013)$. In order to quantify the intermittent structure of response time series, multifractal analyses combine two fundamental classes of analyses: (1) model based analyses of the response time distribution and (2) analyses of the dependency of the time ordering of the responses. Class 1 analyses have shown that the response time distributions across cognitive tasks is unimodal, positively skewed, and with a heavy right tail containing the slow response latencies (e.g., Luce, 1986; Holden et al., 2009). Class 2 analyses have shown that the response times have long-range dependency across hundreds and even thousands of trials and, consequently, that the response time series cannot be considered to be independent random variables assumed by class 1 analyses (Gilden, 2001). The long-range dependency (i.e., monofractal structure) of the response time series are numerical, defined as a single scaling exponent by spectral analyses, autocorrelation analyses, detrended fluctuation analysis, and dispersion analysis, to mention but a few (cf. Diniz et al., 2011). However, Class 2 analyses assume that the response time is Gaussian distributed, whereas Class 1 analyses indicate that they have a nonGaussian heavy tail toward slow response latencies. Multifractal analyses are able to parameterize the non-Gaussian heavy tails that are created using intermittent variation by assessing the complete spectrum of scaling exponents. Thus, multifractal analyses are important extensions of monofractal analyses of response time series.

All multifractal analyses are based on a decomposition of the response time series into a scale-dependent measure that identifies the periods of intermittent variation (see upper panel of Figure 1). The scale dependent measure is the basis for computation of the multifractal spectra along two formalisms (see arrows $\mathrm{A}$ and $B$ in Figure 1). In the Legendre formalism, the scale-dependent measure $\mu_{s, t}$ is used in the computation of the $q$-order moment. $\mu_{s, t}$ is amplified by the positive $q$-orders in the periods with large variation, whereas $\mu_{s, t}$ is amplified by the negative $q$-orders in periods with small variation. An exponent $\zeta_{q}$ is then estimated from the scaling of each of the $q$-order moments before the multifractal spectra are computed from $\zeta_{q}$ (see Ihlen and Vereijken, 2013 for further details). In the large deviation formalism, local exponents are computed from the scaledependent measure $\mu_{s, t}$, and the multifractal spectrum is estimated from the distribution of the local exponents. The increased width of multifractal spectra will reflect more distinct periods of intermittent variation in response time series (see example in Figure 2 in Ihlen and Vereijken, 2013). Additional surrogate tests also detect the periods influenced by multiplicative interactions between temporal scales (Ihlen and Vereijken, 2010). The different multifractal analyses like structure function approach, entropy analyses, wavelet transformation modulus maxima, 


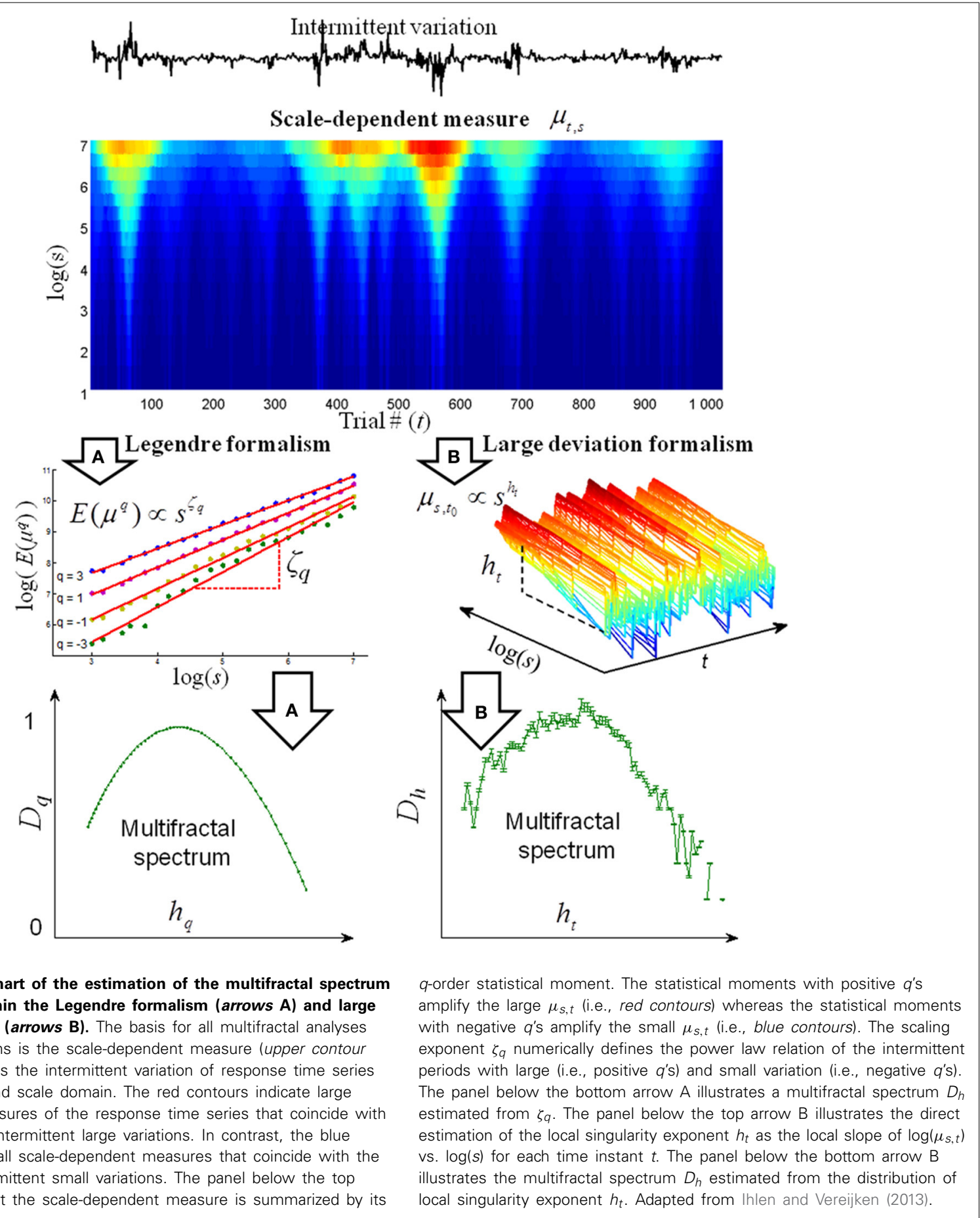

gradient modulus wavelet projection, and multifractal detrended fluctuation analysis are defined by the particular way the scaledependent measures are computed (Ihlen, 2013a; Ihlen and Vereijken, 2013). The Legendre and large deviation formalisms contain statistical assessments of multifractality. Various geometrical assessments have been suggested in the literature that estimates the box counting dimension of the time series (e.g., Russel et al., 1980; Chaudhuri and Sarkar, 1995). However, these methods are only numerically stable for positive $q$ orders and, consequently, only estimate the left tail of the multifractal spectrum. Technical details for the computation of different multifractal analyses within the Legendre and large deviation formalisms, their parameter settings, Matlab codes, and comparison of their performance can be found elsewhere (Kantelhardt et al., 2002; Turiel et al., 2006; Kantelhardt, 2011; Ihlen, 2013a). Multifractal analyses have been applied to several cognitive tasks like simple reaction time, word naming, choice decision, and feedback manipulation (Ihlen and 
Vereijken, 2010; Kuznetsov and Wallot, 2011). All results from these studies indicate that response time series have multifractal properties that are not described by conventional monofractal analyses and that some of these properties might be task dependent.

\section{POTENTIAL PITFALLS IN THE INTERPRETATION OF MULTIFRACTAL ANALYSES}

The interpretation of multifractal spectra of response time series has potential pitfalls. First, the multifractal spectra alone do not indicate that intermittent response time variation is generated by interaction between temporal scales. Wide multifractal spectra of response time series can reflect a power-law response time distribution and not intermittency generated by multiplicative interactions (Ihlen, 2013b). Surrogate tests have to be used to properly identify multiplicative interactions between temporal scales. In these tests, surrogate versions of the response time series are created that eliminate the interaction between temporal scales but preserve all other statistical properties. Multiplicative interaction is present when there is a significant difference between response time series and its surrogate series (e.g., Ihlen and Vereijken, 2010).

Second, response time series of 1000 trials might be too small to establish the presence of multifractality. An ideal monofractal signal will have an infinite number of scales whereas the 1000 trials of response series will only give three scales of order (i.e., 10, 100, and 1000 trials). However, in contrast to ideal monofractal signal, a multifractal signal has scale invariant properties only up to a maximum scale (Bacry et al., 2001). The $q$-order moments and scale-dependent measure converge into a single point on this maximum scale. Thus, in contrast to monofractal analyses, it is sufficient for multifractal analyses to include scales up to the maximum order. Assuming that the signal originates from a prototypical multifractal process, called a multiplicative cascade, the maximum scale could be assessed by analysis of the autocorrelation function (Bacry et al., 2001). Nevertheless, the estimation error of the multifractal spectra related to the number of trials in the response will also be dependent on the chosen $q$-range for the methods within the Legendre formalism and the unknown degree of multifractality. Large degree of multifractality will need large number of trials for a robust assessment of the tails of the multifractal spectra. Consequently, multifractal analysis is quite sensitive to differentiate between monofractal and multifractal response time series, but not between response time series with large degree of multifractality. Furthermore, multifractal analysis of moderately sized response time series will both be more susceptible to noise and non-stationarities compared to longer time series (Ihlen, 2013a). A possible solution is to compare the results of two or more multifractal analysis before interpreting the results. Large deviations in the results of two multifractal analyses indicate that response time series deviate from multifractality and that the results from these analyses must be interpreted with caution.

Third, no single multifractal analysis seems to have superior performance assessing the multifractal spectra of response time series. Previous studies statistical methods based on wavelet transformation, like wavelet transform modulus maxima, has been shown to superior to conventional methods based on the structure function (Muzy et al., 1993). Furthermore, both multifractal detrended fluctuation analysis and gradient modulus wavelet projection has shown superior performance to wavelet transform modulus maxima on moderate sized time series (Kantelhardt et al., 2002; Oświęcimka et al., 2006; Turiel et al., 2006). Kelty-Stephen et al. (2013) have suggested that an entropy based analysis is the best method to assess the multifractal spectrum from response time series and that other multifractal analyses have inferior performance compared to this method using their choice of a scale-dependent measure. However, recent systematic comparison of multifractal analyses shows that all multifractal analyses have different pros and cons and that no single analyses seem to be superior to others (Ihlen, 2013a).

Fourth, the origin of multifractal and intermittent variation in response time series is still debated. Intermittent variation in response time has been suggested to be caused by changes in the participants' attention to stimuli or intermittent error corrections (Ihlen and Vereijken, 2010) and linked to cognitive phenomena like strong anticipation (Stephen and Dixon, 2011). Furthermore, multifractal spectra have been suggested to reflect to a greater extent the presence of self-organization and interaction-dominant dynamics compared to the outcomes of conventional monofractal analyses (Ihlen and Vereijken, 2010; Kelty-Stephen et al., 2013). The interaction-dominant view has been suggested to contrast explicit models of an idiosyncratic mechanism in the cognitive system specific to cognitive tasks or the dynamics of particular localized components (e.g., Van Orden et al., 2003). However, idiosyncratic mechanisms for multifractal variations have been suggested for human locomotion and cardiac function, which indicates that intermittent variations can be generated by task specific components (Ivanov et al., 1998; West and Scafetta, 2003). It is unlikely that any analysis or model will provide conclusive evidence on the generating processes of multifractal variation in response time series (Hasselman, 2013; cf. Kantz and Schreiber, 2004). The generating processes of multifractal and intermittent variation should be decided by experimentation under conditions of strong inference (Hasselman, 2013). Consequently, experimental design should be use to confirm predicted changes in the multifractal spectra. Predicted covariation between local scaling exponents of the response time series and other psychological measures will indicate a common generating process of the multifractality of these signals. As an example, intermittent changes in attention and error correction could be verified by multifractal analyses of gaze fixation and eye movements during the same cognitive task (e.g., Kelty-Stephen and Mirman, 2013).

In summary, caution should be made when inferring response time series as multifractal in a strict mathematical sense. Nevertheless, the width of the multifractal spectra could still be a sensitive index of the intermittency of the response time series even though the intermittency is not prototypical multifractal. The main advantage of multifractal analyses of response time series is their ability to 
assess the temporal changes in their scale invariant structure. Further studies should focus on the assessment of generating processes of multifractal by experimentation under strong inference. This might include the assessment of temporal changes in the local scaling exponent (i.e., the local structure of response time variation) in more heterogeneous and real-life experiments where the task conditions and characteristics of the stimuli involve change across trials. Furthermore, the correlation between the temporal changes in the structure of the response time variation and other neurophysiological and psychological measurements can be assessed through multifractal analyses by correlating the temporal change of the scaling exponents (see example in Figure 7 in Ihlen and Vereijken, 2013). Time series from different levels of the cognitive and neurophysiological system are more likely to correlate in their scale independent structure rather than their unit dependent magnitude. Thus, multifractal analyses might provide new insight into the interaction and coordination of multiple levels of cognitive performance and human behavior.

\section{REFERENCES}

Bacry, E., Delour, J., and Muzy, J. F. (2001). Multifractal random walk. Phys. Rev. E 64:026103. doi: 10.1103/PhysRevE.64.026103

Chaudhuri, B., and Sarkar, N. (1995). Texture segmentation using fractal dimension. IEEE Trans. Pattern Anal. Mach. Intell. 17, 72-77. doi: $10.1109 / 34.368149$

Diniz, A., Wijnants, M. L., Torre, K., Barreiros, J., Crato, N., Bosman, A. M. T., et al. (2011). Contemporary theories of $1 / \mathrm{f}$ noise in motor control. Hum. Mov. Sci. 30, 889-905. doi: 10.1016/j.humov.2010.07.006

Gilden, D. L. (2001). Cognitive emission of $1 / \mathrm{f}$ noise. Psychol. Rev. 108, 33-56. doi: 10.1037/0033295X.108.1.33

Hasselman, F. (2013). When the blind curve is finite: dimension estimation and modell inference base don empirical waveforms. Front. Physiol. 4:75. doi: 10.3389/fphys.2013.00075
Holden, J. G., Van Orden, G. C., and Turvey, M. T. (2009). Dispersion of response times reveals cognitive dynamics. Psychol. Rev. 116, 318-342. doi: 10.1037/a0014849

Ihlen, E. A. F. (2013a). Multifractal analyses of response time series: a comparative study. Behav. Res. 45, 928-945. doi: 10.3758/s13428-013-0317-2

Ihlen, E. A. F. (2013b). The influence of power law distributions on long-range trial dependency of response times. J. Math. Psychol. 57, 215-224. doi: 10.1016/j.jmp.2013.07.001

Ihlen, E. A. F., and Vereijken, B. (2010). Interaction dominant dynamics in human cognition: beyond $1 / f^{\alpha}$ fluctuations. J. Exp. Psychol. Gen. 139, 436-463. doi: 10.1037/a0019098

Ihlen, E. A. F., and Vereijken, B. (2013). Multifractal formalisms of human behaviour. Hum. Mov. Sci. 32, 633-651. doi: 10.1016/j.humov.2013.01.008

Ivanov, P. C., Amaral, L. A. N., Goldberger, A. L., and Stanley, H. E. (1998). Stochastic feedback and the regulation of biological rhytms. Europhys. Lett. 43 , 363-368. doi: 10.1209/epl/11998-00366-3

Kantelhardt, J. W. (2011). "Fractal and multifractal time series," in Mathematics of Complexity and Dynamical Systems, ed R. A. Meyers (New York, NY: Springer), 463-487.

Kantelhardt, J. W., Zschiegner, S. A., Koscielny-Bunde, E., Havlin, S., Bunde, A., and Stanley, H. E. (2002). Multifractal detrended fluctuation analysis of nonstationary time series. Phys. A 316, 87-114. doi: 10.1016/S0378-4371(02)01383-3

Kantz, H., and Schreiber, T. (2004). Nonlinear Time Series Analysis. Cambridge: Cambridge University Press.

Kelty-Stephen, D. G., and Mirman, D. (2013). Gaze fluctuations are not additively decomposable: reply to Bogartz \& Staub. Cognition 126, 128-134. doi: 10.1016/j.cognition.2012.09.002

Kelty-Stephen, D. G., Palatinus, K., Saltzman, E., and Dixon, J. A. (2013). A tutorial on multifractality, cascades, and interactivity for empirical time series in ecological science. Ecol. Psychol. 25, 1-62. doi: 10.1080/10407413.2013.753804

Kuznetsov, N. A., and Wallot, S. (2011). Effects of accuracy feedback on fractal characteristics of time estimation. Front. Integr. Neurosci. 5:62. doi: $10.3389 /$ fnint.2011.00062

Luce, R. D. (1986). Response Times: Their Role in Inferring Elementary Mental Organization. Oxford: Oxford University Press.

Mandelbrot, B. B. (1974). Intermittent turbulence in self-similar cascades: divergence of high moments and dimension of the carrier. J. Fluid Mech. 62, 331-358. doi: 10.1017/S0022112074000711

Muzy, J. F., Bacry, E., and Arneodo, A. (1993). Multifractal formalism for fractal signals: the structure function approach versus the wavelettransform modulus-maxima method. Phys. Rev. E 47, 875-884. doi: 10.1103/PhysRevE. 47.875

Oświęcimka, P., Kwapien, J., and Drozdz, S. (2006). Wavelet versus detrended fluctuation analysis of multifractal structures. Phys. Rev. E 74:016103. doi: 10.1103/PhysRevE.74.016103

Russel, D., Hanson, J., and Ott, E. (1980). Dimension of strange attractors. Phys. Rev. Lett. 45, 1175-1178. doi: 10.1103/PhysRevLett.45.1175

Stephen, D. G., and Dixon, J. A. (2011). Strong anticipation: multifractal cascade dynamics modulate scaling in synchronization behaviors. Chaos Solit. Fract. 44, 160-168. doi: 10.1016/j.chaos.2011.01.005

Turiel, A., Perez-Vicente, C. J., and Grazzini, J. (2006). Numerical methods for the estimation of the estimation of the multifractal singularity spectra on sampled data: a comparative study. $J$. Comp. Phys. 216, 362-390. doi: 10.1016/j.jcp.2005. 12.004

Van Orden, G. C., Holden, J. G., and Turvey, M. T. (2003). Self-organization of cognitive performance. J. Exp. Psychol. Gen. 132, 331-350. doi: 10.1037/0096-3445.132.3.331

West, B. J., and Scafetta, N. (2003). Nonlinear dynamical model of human gait. Phys. Rev. E 67:051917. doi: 10.1103/PhysRevE.67.051917

Yaglom, A. M. (1966). Effect of fluctuations in energy dissipation rate on the form of turbulence characteristics in the inertial subrange. Dokl. Akad. Nauk SSSR 166, 49-52.

Conflict of Interest Statement: The author declares that the research was conducted in the absence of any commercial or financial relationships that could be construed as a potential conflict of interest.

Received: 23 April 2014; accepted: 27 June 2014; published online: 21 July 2014.

Citation: Ihlen EAF (2014) Multifractal analyses of human response time: potential pitfalls in the interpretation of results. Front. Hum. Neurosci. 8:523. doi: 10.3389/fnhum.2014.00523

This article was submitted to the journal Frontiers in Human Neuroscience.

Copyright $(\odot 2014$ Ihlen. This is an open-access article distributed under the terms of the Creative Commons Attribution License (CC BY). The use, distribution or reproduction in other forums is permitted, provided the original author(s) or licensor are credited and that the original publication in this journal is cited, in accordance with accepted academic practice. No use, distribution or reproduction is permitted which does not comply with these terms. 